\title{
NEUMONÍA E INSUFICIENCIA CARDIACA, MÁS ALLÁ DE UN SENTIDO BIDIRECCIONAL
}

\author{
PNEUMONIA AND HEART FAILURE, BEYOND A BIDIRECTIONAL SENSE \\ Paola L. A. Oruro-Cari ${ }^{1,2}$
}

\section{Sr. Editor}

La neumonía adquirida en la comunidad (NAC) sigue siendo un tema en voga, siendo definida como un trastorno inflamatorio agudo del parénquima pulmonar en pacientes que han adquirido la infección en la comunidad y no han sido hospitalizados durante las últimas 3 semanas'.

La NAC es la enfermedad infecciosa más letal a nivel mundial y su creciente incidencia es consecuente al aumento de enfermedades crónicas, inmunosupresión y esperanza de vida, que conlleva a un aumento de población vulnerable ${ }^{2}$.

Por otro lado, la insuficiencia cardiaca (IC) es, junto con otras enfermedades cardiovasculares (ECV), la primera causa de muertes en el mundo ${ }^{3}$. Las enfermedades cardiovasculares ocupan el cuarto lugar en el Perú4.

En la guía de práctica clínica de NAC de la Sociedad Peruana de Enfermedades Infecciosas y Tropicales, la insuficiencia cardiaca figura entre los factores de riesgo de NAC, tabla $1^{1}$. Sin embargo, existe controversia acerca de la dirección de la relación causal de insuficiencia cardíaca (IC) frente a neumonía adquirida en la comunidad.

Esta ambivalencia recae sobre si la IC conlleva a un inmuno-compromiso que ofrece una puerta de entrada a los agentes de infección, o si la NAC se presenta como un factor asociado al desarrollo de enfermedad cardiovascular. Frente a esta última opción, Corrales et al. sugieren algunas repercusiones de la neumonía aguda sobre el sistema cardiovascular, tabla $2^{5}$.

Tabla 1. Factores de riesgo asociados a neumonía adquirida en la comunidad.

\section{FACTORES DE RIESGO}

Enfermedad estructural bronquial y/o parenquimal

EPOC $^{1}$

Bronquiectasias

Inmunodeficiencia congénita o adquirida

Tabaquismo

Alcoholismo

Infecciones respiratorias virales recientes

Insuficiencia cardiac

Terapia inmunosupresora

Hepatopatías crónicas

Neoplasias

Exposición a temperaturas bajas ${ }^{2}$

1 EPOC: Enfermedad pulmonar obstructiva crónica

2 Sobre todo en ancianos

${ }^{1}$ Facultad de Medicina, Universidad Nacional de San Agustín, Arequipa-Perú.

a Estudiante de Medicina.

Citar como: Paola L. A. Oruro-Cari. Neumonía e insuficiencia cardiaca, más allá de un sentido bidireccional. [Cartas al Editor].2019;19(2):00-00. (Enero 2018). DOI 10.25176/RFMH.v19.n2.2073

( ) Los autores. Este artículo es publicado por la Revista de la Facultad de Medicina Humana, Universidad Ricardo Palma. Este es un artículo de Open Access distribuido bajo los términos de la Licencia Creative Com- mons: Creative Commons Attribution 4.0 International(CC BY 4.0). (https://creativecommons.org/licenses/by/4.0/), que permite el uso no comercial, distribución y reproducción en cualquier medio, siempre que la obra original sea debidamente citadas. Para uso comercial, por favor póngase en contacto con revista.medicina@urp.pe 
Tabla 2. Efectos de Neumonía Aguda sobre el sistema cardiovascular.

\section{EFECTOS EN COMPONENTES CARDIOVASCULARES}

Endotelio

Miocardio

Ritmo cardiaco

Arterias coronarias

Circulación pulmonar

Función autonómica cardiaca

\section{Coagulación}

Función renal y osmoesquilibrio

NA: Neumonía Aguda

RVP: Resistencia vascular periférica

AVP: Arginina vasopresina

ECA: Enzima convertidora de angiotensina

En la guía de práctica clínica de cardiología del 2013, publicada por el ministerio de salud, no se considera este factor de asociación a IC, tabla $3^{6}$.

Un evento cardiovascular en el curso de NAC da paso a un mal pronóstico. En una revisión de Cillóniz et al. se registra que aproximadamente la quinta parte de los casos están asociados a complicaciones cardiovasculares y la IC está entre las razones más comunes de readmisión ${ }^{7}$. Esto puede deberse a las complicaciones a las que se presta la enfermedad, como concuerda Violi et al. en cuyo estudio la mortalidad de pacientes con NAC fue mayor $(17,6 \%)$ en pacientes que desarrollaron ECV frente a los que no $(4,5 \%)^{8}$. Aunque en otro estudio la edad y la comorbilidad no estaban asociados a NAC en los ingresos hospitalarios y solo la edad lo estaba a las defunciones por la misma causa ${ }^{9}$. A su vez, Feldman et al. indican que un $30 \%$ de pacientes hospitalizados por NAC llegan a desarrollar un $\mathrm{ECV}^{10}$. Sin embargo, hablamos de eventos a corto plazo.

Tabla 3. Factores de riesgo asociados a insuficiencia cardiaca.

\section{MEDIO AMBIENTE}

No aplica

\section{ESTILOS DE VIDA}

\section{Obesidad}

Inactividad Física

Dieta aterogénica

\section{FACTORES HEREDITARIOS}

Historia familiar de enfermedad coronaria prematura

\begin{tabular}{|c|c|c|}
\hline Enfermedad coronaria en & Varones & 55 años \\
\hline familiares de primer grado & Mujeres & 65 años \\
\hline
\end{tabular}


Un estudio de cohorte en Canadá demostró que la neumonía adquirida en la comunidad aumenta sustancialmente el riesgo de insuficiencia cardíaca a largo plazo, de manera que los pacientes con NAC de edad menor o igual a 65 años tienen 1,98 veces más probabilidad de desarrollar IC a diferencia de los pacientes mayores de 65 años cuyo riesgo relativo es más bajo. Asimismo, incrementa la probabilidad de ser hospitalizado por un incidente relacionado a insuficiencia cardiaca al año del alta médica $(R R=1.86$, 1.50 a $2.32, \mathrm{p}<0.001)^{11}$.

A pesar de que la población por debajo de los 65

Correspondencia: Paola Lesly Asunción Oruro Cari

Dirección: Calle JS Chocano 104 Urb. 15 de Enero - Paucarpata, Arequipa - Perú.

Celular: 965707068

Correo:paoola.soad79@gmail.com

\section{REFERENCIAS BIBLIOGRÁFICAS}

1. Sociedad Peruana de enfermedades infecciosas y tropicales. Guía de práctica clínica Neumonía adquirida en la Comunidad en Adultos. Perú, Organización Panamericana de la salud, 2009. [Citado 2019 Feb 14]. Disponible en: bvs.minsa.gob.pe/local/minsa/2418.pdf

2. Cillóniz C, Cardozo C, García-Vidal C. Epidemiology, pathophysiology, and microbiology of communityacquired pneumonia. Ann Res Hosp [Internet] 2018 [cited 2019 Feb 12];2(1). Disponible en: http://arh.amegroups.com/ article/view/3921/4657

3. American Heart Association. Resumen de estadísticas de 2017 Enfermedad del corazón y ataque cerebral [Internet]. 2017 [cited 2019 Feb 14]. Disponible en: https://professional.heart.org/idc/groups/ahamahpublic/@wcm/@sop/@smd/documents/downloadable/ucm_491392.pdf

4. Ministerio de salud. Dirección General de Epidemiología. Análisis de la Situación de Salud del Perú [Internet]. 2010 [cited 2019 Feb 17] 51-78. Disponible en: www.inei.gob.pe/media/MenuRecursivo/publicaciones digitales/Est/Lib1146/cap02.pdf

5. Corrales VF, Musher DM, Shachkina S, Chirinos JA. Acute pneumonia and the cardiovascular system. Lancet [Internet]. 2013 Feb [cited 2019 Feb 14];381 (9865):496-505. Disponible en: https://linkinghub.elsevier.com/ retrieve/pii/S0140673612612665

6. Departamento de especialidades médicas. Guía de práctica clínica de cardiología. Lima, Instituto nacional de enfermedades neoplásicas, 2013. [Citado 2019 Feb 14]. Disponible en: www.inen.sld.pe/portal/ años no es la más propensa a contraer neumonía adquirida en la comunidad, no considerar la neumonía como factor de riesgo de IC expondría a la población económicamente activa (PEA) a eventos cardiovasculares que lleven a más pérdida de productividad de la que estamos viviendo con la incidencia actual de ECV.

Por ello, se propone que en la próxima actualización de las guías de manejo clínico se evalúe a la neumonía como comorbilidad de IC enfatizando la población adulta menor de 65 años como vulnerable a largo plazo y promover estrategias preventivas del caso.
documentos/pdf/normas_tecnicas/2013/03072014_GU\%C3\%8DA_DE PR\%C3\%81CTICA_CL\%C3\%8DNICA_DE_CARDIOLOG\%C3\%8DA.pdf.

7. Cillóniz C., Rodríguez D., Nicolini A.et al. SYMPOSIUM REVIEW ARTICLE: Clinical Approach to Community-acquired Pneumonia. J Thorac Imaging 2018;33(5):273-281. [Citado 2019 Feb 14]. Disponible en: Insights.ovid.com/ crossref?an=00005382-900000000-99581.

8. Violi F. et al. Cardiovascular Complications and Short-term Mortality Risk in Community-Acquired Pneumonia. Clinical Infectious Diseases, [Internet]. 2017 [citado 2019 Feb 18]; 64(1):1486-1493. Disponible en: academic.oup. com/cid/article-lookup/doi/10.1093/cid/cix164.

9. Irizar Aramburu Ml, Arrondo Beguiristain MA, Insausti Carretero MJ, Mujica Campos J, Etxabarri Perez P, Ganzarain Gorosabel R. Epidemiología de la neumonía adquirida en la comunidad. Atención Primaria [Internet]. Elsevier; 2013 Dec 1 [cited 2019 Feb 12];45 (10):503-13. Disponible en: https://linkinghub.elsevier.com/retrieve/pii/S0212656713001625.

10. Feldman C. et al. Pathogenesis and prevention of risk of cardiovascular events in patients with pneumococcal community-acquired pneumonia. Journal of Internal Medicine. [Internet]. 2018 [cited 2019 Feb 18], 1(1) Disponible en: https://onlinelibrary.wiley.com/doi/full/10.1111/joim.12875.

11. Eurich D, Marrie T, Minhas-Sandhu J. Risk of heart failure after community acquired pneumonia: prospective controlled study with 10 years of followup.BMJ [Internet]. 2017 Ene [cited 2019 Feb 14]. Disponible en: www.bmj. com/lookup/doi/10.1136/bmj.j413. 